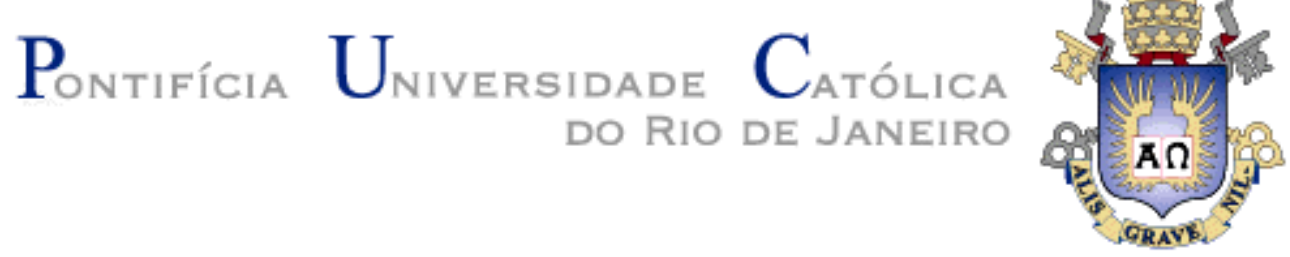

Bernardo Antônio Almeida Pinto de Souza

Arquétipos do Pai: A Paternidade e o Masculino em Transformação

Dissertação de Mestrado

Dissertação apresentada ao Programa de PósGraduação em Psicologia Clínica da PUC-Rio como requisito parcial para obtenção do grau de Mestre em Psicologia.

Orientadora: Profa. Lídia Levy de Alvarenga 


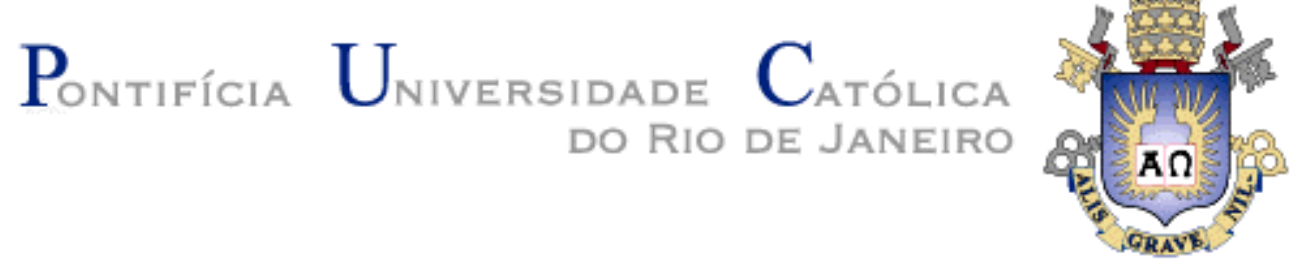

Bernardo Antônio Almeida Pinto de Souza

\section{Arquétipos do Pai: A Paternidade e o Masculino em Transformação}

Dissertação apresentada como requisito parcial para obtenção do grau de Mestre pelo Programa de PósGraduação em Psicologia Clínica da PUC-Rio. Aprovada pela Comissão Examinadora abaixo assinada.

Profa. Lídia Levy de Alvarenga

Orientadora

Departamento de Psicologia Clínica - PUC-Rio

Prof. Walter Fonseca Boechat

Universidade Estadual do Rio de Janeiro - UERJ

Prof. Álvaro de Pinheiro Gouvêa Departamento de Psicologia Clínica - PUC-Rio

Profa. Denise Berruezo Portinari

Coordenadora Setorial do Centro de Teologia e Ciências Humanas - PUC-Rio

Rio de Janeiro, 19 de março de 2014 
Todos os direitos reservados. É proibida a reprodução total ou parcial do trabalho sem autorização do autor, da orientadora e da universidade.

\section{Bernardo Antônio Almeida Pinto de Souza}

Graduou-se em Psicologia na UFRJ (Universidade Federal do Rio de Janeiro) em 2012. Pós-Graduado no Curso de Especialização em Psicologia Junguiana na Universidade Estácio de Sá. Participa do Núcleo-Rio da Abrapso, no qual pesquisa acerca das relações entre psicologia e educação no Brasil. Atua como psicólogo clínico. Temas de interesse: psicologia social, psicologia analítica, articulações entre psicologia e mitologia, articulações entre psicologia e histórias em quadrinhos e questões acerca da paternidade e masculinidade no mundo contemporâneo.

Ficha Catalográfica

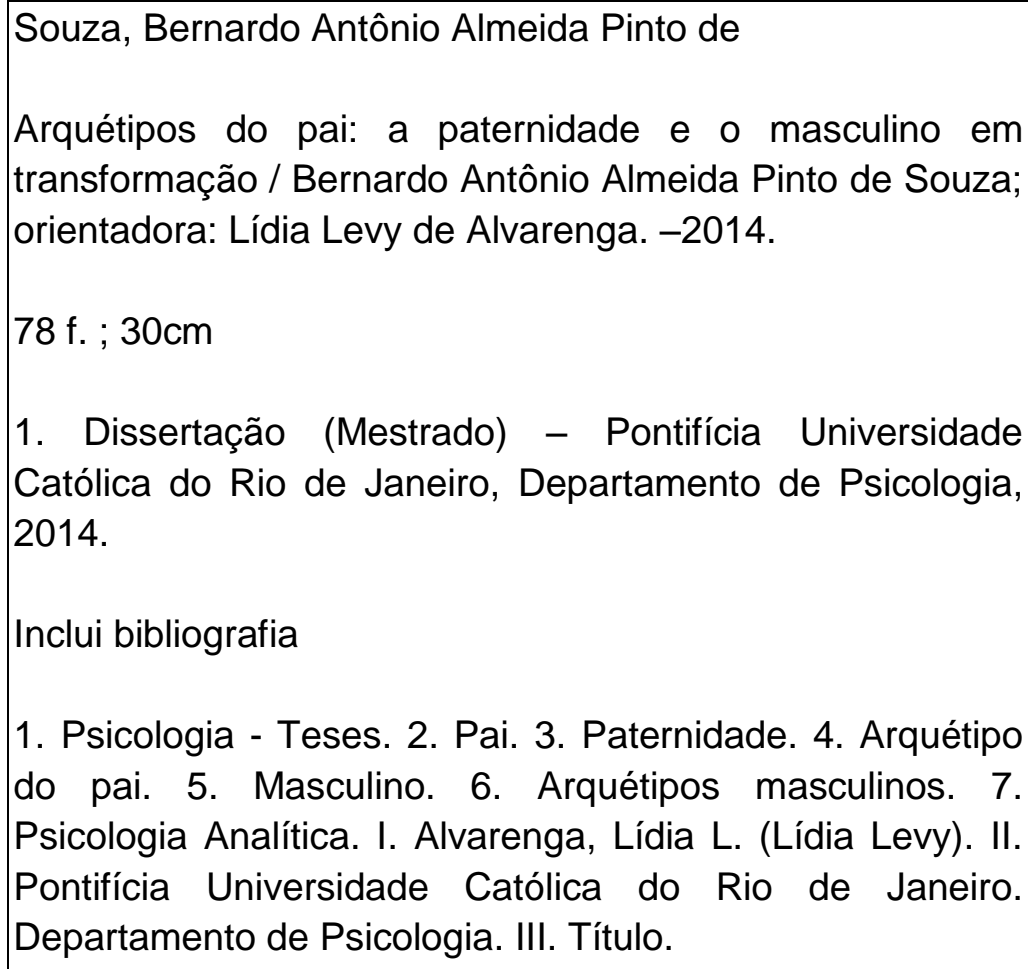
transformação / Bernardo Antônio Almeida Pinto de Souza; orientadora: Lídia Levy de Alvarenga. -2014.

78 f. ; $30 \mathrm{~cm}$

1. Dissertação (Mestrado) - Pontifícia Universidade Católica do Rio de Janeiro, Departamento de Psicologia, 2014.

Inclui bibliografia

1. Psicologia - Teses. 2. Pai. 3. Paternidade. 4. Arquétipo do pai. 5. Masculino. 6. Arquétipos masculinos. 7. Psicologia Analítica. I. Alvarenga, Lídia L. (Lídia Levy). II. Pontifícia Universidade Católica do Rio de Janeiro. Departamento de Psicologia. III. Título. 


\section{Agradecimentos}

À Lidia Levy de Alvarenga, por me receber na PUC, pela orientação e observações minuciosas no percurso deste trabalho.

Ao professor Walter Fonseca Boechat, pela participação na banca, pelo auxílio com a confecção do trabalho e pelas indicações bibliográficas.

Ao professorÁlvaro de Pinheiro Gouvêa por participar da banca e da avaliação deste trabalho.

À Leila Sanches de Almeida, cuja pesquisa despertou meu interesse pelo tema.

À Adriana Carrijo, pelo auxílio constante e pela amizade.

A CAPES e à PUC-Rio, pelos auxílios concedidos, sem os quais este trabalho não poderia ter sido realizado.

À minha família (Ana, Marcos e Gabriela), por estarem sempre presentes.

À Flávia pelo auxílio na revisão do texto, pelo convívio durante a construção deste trabalho, pelo amor compartilhado e pela força que me proporcionou. 


\section{Resumo}

Souza, Bernardo Antônio Almeida Pinto de; Alvarenga, Lídia Levy de. Arquétipos do Pai: A Paternidade e o Masculino em Transformação. Rio de Janeiro, 2014. 78p. Dissertação de Mestrado - Departamento de Psicologia, Pontifícia Universidade Católica do Rio de Janeiro.

Na composição familiar, o papel do pai foi um dos que mais se transformou ao longo dos últimos anos. Nesta dissertação, propomos uma investigação acerca das mudanças pelas quais passaram e ainda passam os homens e pais na pósmodernidade. Para tanto, nos servimos do arcabouço teórico da Psicologia Analítica de C. G. Jung. Dentro desta linha teórica, destacamos principalmente os arquétipos masculinos e o arquétipo do pai, os quais muitas vezes se confundem, bem como suas manifestações diferenciadas nos dias de hoje. De modo análogo, ponderamos os estatutos da persona e da sombra no pai da modernidade e no pai da atualidade. A ideia de pai já existia de forma inconsciente antes do entendimento da paternidade física. A paternidade, entendida como uma invenção social, é uma atribuição específica do homem, enquanto a maternidade é específica da mulher. Foi necessário fazer o contraponto entre masculino e feminino, entre patriarcado e matriarcado, assim como paternidade e maternidade, pois tais temas se mostram profundamente entrelaçados. Além disso, recorremos à mitologia e aos rituais descritos em estudos antropológicos para auxiliar no entendimento da antiguidade destas questões. Haja vista a ocorrência de uma relativização dos arquétipos masculinos na cultura atual, propomos a discussão do conceito de "novo pai" e se este poderia ser considerado como um novo símbolo do masculino no campo da paternidade.

\section{Palavras-chave}

Pai; paternidade; arquétipo do pai; masculino; arquétipos masculinos; Psicologia Analítica. 


\section{Abstract}

Souza, Bernardo Antônio Almeida Pinto de; Alvarenga, Lídia Levy de (Advisor). Archetypes of the Father: The Paternity and Masculine in Transformation. Rio de Janeiro, 2014. 78p. MSc. Dissertation Departamento de Psicologia, Pontifícia Universidade Católica do Rio de Janeiro.

In family composition, the role of the father was one of the most has been transformed over the past few years. In this dissertation, we propose an investigation into the changes that have gone and still go men and fathers in postmodernity. In order to do this, we took the theoretical framework of the Analytical Psychology of C.G. Jung. Within this theoretical line, we include mainly masculine archetypes and archetype of the father, which often overlap, as well as their different manifestations today. Similarly, we ponder the statutes of the persona and the shadow of his father and the father of modernity today. The idea of father has existed before unconsciously understanding of physical paternity. Fatherhood, understood as a social invention is a specific allocation of man, while maternity is specific of the woman. It was necessary to make the contrast between masculine and feminine, between patriarchy and matriarchy, between fatherhood and motherhood, because such issues are deeply intertwined show. Furthermore, we resort to the mythology and rituals described by the anthropological studies to facilitate the understanding of ancient studies of these issues. Given the occurrence of a relativization of masculine archetypes in the current culture, we propose to discuss the concept of "new father" and whether this could be considered as a new symbol of the masculine in the field of fatherhood.

\section{Keywords}

Father; paternity; father archetype; masculine; masculine archetypes; Analytical Psychology. 


\section{Sumário}

1. Introdução 9

2. Aspectos teóricos da Psicologia Analítica $\quad 17$

2.1. Carl Gustav Jung e a Psicologia Analítica 17

2.2. A equação pessoal e a psicologia científica 18

2.3. Teste de associação de palavras e complexos 20

2.4. O estudo da mitologia 22

2.5. Duas formas de pensamento e tipos psicológicos 25

2.6. Símbolos 26

2.7. Arquétipos e inconsciente coletivo $\quad 27$

2.8. Anima e animus 28

2.9. Persona e sombra $\quad 29$

2.10. Si-mesmo (Self) 32

2.11. Individuação 32

3. O arquétipo do pai $\quad 34$

3.1. O pai para Jung 34

3.2. As funções céu e terra 36

3.3. Menino e homem 39

3.4. Outras perspectivas de arquétipo do pai 41

4. A paternidade em transformação 43

4.1. Patriarcado e matriarcado 43

4.2. Masculino e feminino $\quad 45$

4.3. Paternidade e maternidade 53

5. O pai da pós-modernidade 59

5.1. Uma breve incursão pela modernidade: a constituição de um
novo modelo familiar

5.2. A crise de identidade masculina na pós-modernidade 61

5.3. A relativização dos arquétipos masculinos na cultura atual 64

$\begin{array}{ll}\text { 5.4. Um "novo pai"? } & 67\end{array}$

$\begin{array}{ll}\text { 6. Considerações finais } & 71\end{array}$

$\begin{array}{ll}\text { 7. Referências bibliográficas } & 75\end{array}$ 